Research paper

\title{
Inhibitors to factor VII and in congenital factor VII deficiency
}

\author{
Angelika Batorova, ${ }^{1}$ Guglielmo Mariani, ${ }^{2}$ Kaan Kavakli, ${ }^{3}$ Arlette Ruiz de Saez, \\ Ümran Caliskan, ${ }^{5}$ Mehran Karimi, ${ }^{6}$ Mirko Pinotti, ${ }^{7}$ Mariasanta Napolitano, ${ }^{8}$ Alberto \\ Dolce, ${ }^{9}$ Benny Sørensen ${ }^{10}$ and Jørgen Ingerslev, ${ }^{11}$ on behalf of the STER Study Group
}

${ }^{1}$ The National Haemophilia Centre, Institute of Haematology and Blood Transfusion, University Hospital, Bratislava, Slovakia, ${ }^{2}$ University of Ferrara Medical School, Ferrara, Italy, ${ }^{3}$ Department of Paediatric Haematology, Ege University Hospital, Ege Haemophilia Center, Izmir, Turkey, ${ }^{4}$ National Haemophilia Centre, Banco Municipal de Sangre DC, Caracas, Venezuela, ${ }^{5}$ Department of Paediatric Haematology, Necmettin Erbakan University, Konya, Turkey, ${ }^{6}$ Shiraz University of Medical Sciences, Haematology Research Center, Shiraz, Iran, ${ }^{7}$ Department of Life Sciences and Biotechnology, University of Ferrara, Ferrara, Italy, ${ }^{8}$ University of Palermo, Haematology Unit, Thrombosis and Hemostasis Reference Regional Center, Palermo, Italy, ${ }^{9}$ National Institute of Statistics, Palermo, Italy, ${ }^{10}$ Haemostasis and Thrombosis Centre, Guy's \& St Thomas' Hospital, London, UK and ${ }^{11}$ Centre for Haemophilia and Thrombosis, Aarhus University Hospital, Skejby, Aarhus, Denmark

Correspondence: Guglielmo Mariani, University of Ferrara, Ferrara, Italy. e-mail: gmprivate39@gmail.com

Running title: FVII inhibitors in congenital FVII deficiency: the STER [55 characters; maximum 60 characters and spaces] 


\section{Summary}

Congenital factor VII (FVII) deficiency is a rare bleeding disorder, and few anecdotal reports are available regarding FVII inhibitors. Using data from the Seven Treatment Evaluation Registry (STER; 225 patients, 312 treatments), we have prospectively evaluated the occurrence of FVII inhibitors using a standardized method over a period of 8 years. The central laboratory screened 115 paired samples; FVII inhibitors were detected in $3 / 115$ (2.6\%) patients (one de novo inhibitor and two pre-existing inhibitors). A fourth inhibitor was detected in a patient who had been screened locally. All four patients had high-responding inhibitors (10-72 BU) and had previously received factor replacement therapy. In three patients, the inhibitors appeared before the age of 6 months during prophylaxis following central nervous system or gastrointestinal bleeds; however, treatments were continued without apparent loss of efficacy. In one patient, an inhibitor developed in adulthood after replacement therapy for minor surgery. No anaphylactoid reactions or renal complications were reported during or after prophylaxis. In conclusion, inhibitor development is rare in patients with congenital FVII deficiency $(\sim 2 \%)$, with an incidence similar to that in patients with haemophilia B. FVII inhibitors display immunological (anamnesis) and kinetic features similar to those in patients with haemophilia.

Summary word count: 198 words (maximum 200 words)

Keywords: Congenital FVII deficiency, factor VII, FVII inhibitors, registry, STER.

Clinicaltrials.gov identifier: NCT01269138 


\section{Introduction}

Antibodies to clotting factors have been consistently reported in the medical literature; the first was described in a patient with haemophilia A treated numerous times with blood products (Lawrence \& Johnson, 1941). These antibodies are considered 'allo-antibodies' if they arise after the administration of a therapeutic protein during replacement therapy in patients with congenital bleeding disorders, or 'auto-antibodies' if a congenital bleeding disorder is not present. The latter usually occur in the elderly and can be associated with a variety of comorbidities. Clinically, only those antibodies that affect clotting activity are termed 'inhibitors' and are therefore considered relevant, because they may render patients refractory to treatment.

In patients with haemophilia A or B, inhibitors can occur after replacement therapy with factor VIII (FVIII) or factor IX (FIX) concentrates, respectively. The risk of developing inhibitors is particularly high in patients with severe deficiencies (FVIII or FIX $<1 \%$ of normal) and in those with null mutations (Coppola et al, 2010; Oldenburg \& Pavlova, 2006). Furthermore, inhibitors are far more frequent in patients with haemophilia A (typically 15-20\%; range, 8-52\%) than in patients with haemophilia B ( 1-3\%) (DiMichele, 2007; Mariani et al, 2012a). This difference is considered attributable, at least in part, to the larger size and complexity of FVIII compared with FIX. In any event, the ensuing immunological triggers and mechanisms associated with inhibitor development are not yet fully understood.

The development of an inhibitor to a clotting factor is associated with increased rates of morbidity and mortality, an increased cost of care and more complicated treatment regimens (Bolton-Maggs, 2006; Franchini et al, 2013). When compared with haemophilia A, the immune response in haemophilia B has several distinctive features: (i) an increased risk of developing anaphylaxis after the administration of FIX-containing concentrates to a patient with a FIX inhibitor; (ii) a risk of developing nephrotic syndrome after exposure to FIX replacement products; and (iii) a lower success rate of immune tolerance therapy for the eradication of FIX inhibitors (Coppola et al, 2010; DiMichele, 2012).

Congenital FVII deficiency is a rare bleeding disorder and there are few data regarding FVII inhibitors. FVII shares considerable homology with FIX, both at the gene and protein levels (Furie \& Furie, 1992), and displays a comparable distribution of disease- 
causing mutations, with a large predominance of missense changes (Bernardi et al, 2009). Anecdotal reports of inhibitors to FVII have been previously published in the literature, including reports from members of our group (Nicolaisen et al, 1996; Mariani et al, 1999; Ingerslev et al, 2005; Pruthi et al, 2007, Batorova et al, 2007; Tokgoz et al, 2012). Here, we report a prospective study in which screening for FVII antibodies was performed according to a specific protocol within the frame of the Seven Treatment Evaluation Registry (STER), which also collected data on the treatment of spontaneous bleeding episodes, surgical interventions and prophylaxis in patients with congenital FVII deficiency over an 8-year period (Mariani et al, 2011; Mariani et al, 2012b; Mariani et al, 2013; Napolitano et al, 2013). The inhibitor screening procedure was carried out centrally and data were analyzed in a standardized clinical and laboratory context (Ingerslev et al, 2005).

\section{Methods}

\section{STER database}

The STER (www.targetseven.org) is a multicentre, prospective, observational, Web-based registry that collected structured and detailed data on the management of FVII deficiency. STER followed the strictly controlled data collection procedures established by the International FVII Deficiency Study Group (IF7SG) (Bernardi et al, 2009; Mariani et al, 2005; Mariani et al, 2011; Mariani et al, 2012b; Mariani et al, 2013; Mariani \& Bernardi, 2009; Napolitano et al, 2013). The STER protocol is published on clinicaltrials.gov (NCT01269138). Blood samples for centralized inhibitor determination (Ingerslev et al, 2005) were collected at enrolment, $30 \mathrm{~d}$ following administration of one or more replacement therapies, and as needed to check titres after multiple administrations at other times. Clinical and laboratory information was collected using Web-based forms and stored in a Siemens mainframe. Another form captured previous treatments and the actual regimen adopted (type of product, dosage and schedule), treatment efficacy, complications and adverse events. Investigators were also asked to report any bleeding episodes that occurred during the observation period.

The registry database was closed on 28 February 2012, by which time all inhibitor assays had been completed, and the data were checked for consistency. 


\section{Plasma collection and laboratory procedures}

Platelet-free plasma samples were collected locally by a contract research organization, centrifuged, transferred to a repository, and stored at $-80^{\circ} \mathrm{C}$ before being sent on dry ice to the central laboratory. Provisions were made to ensure that delivery of each shipment was pre-announced and at receipt, and that all samples were stored at $-80^{\circ} \mathrm{C}$ until analysis, which occurred within four weeks. Each sample was assayed for FVII:C; assays were performed on the Thrombolyzer instrument (Benkh, Norderstedt, Germany). No clinical data were submitted to the central laboratories that received the plasma samples.

Inhibitors to FVII were detected using a modified Bethesda Assay that used a highsensitivity thromboplastin as an 'activator' (Innovin, Siemens Diagnostica, Germany) (Ingerslev et al, 2005). The FVII-deficient substrate plasma was a natural deficient plasma obtained from a severely affected patient (FVII:C $<1 \%$; homozygous Arg100GLN mutation), and the diluent was an Imidazole buffer (50 mmol, $\mathrm{pH} 7.3)$. The calibrator was a Verify Reference Plasma (Lot 113029, Organon-Teknika, Durham, NC, USA) with an assigned FVII:C value of $1.12 \mathrm{IU} / \mathrm{ml}$. The 1:1 mixture incubation period was 2 hours. An inhibitor titre of $<0.6 \mathrm{BU}$ was considered negative. Results were considered valid if the coefficient of variation (CV) of double determinations did not exceed $25 \%$ and if at least two diluted sample results were between $25 \%$ and $75 \%$ of the reference sample result. From the beginning of the study until May 2010, samples were screened at the Skejby Haemophilia and Thrombosis Laboratory, Skejby University Hospital, Denmark; after that date, and until February 2012, samples were screened at the St. Thomas' Central Laboratory, London, UK, using the same protocol and laboratory procedures. Only plasma samples with phenotypic baseline FVII:C levels at $<4 \%$ of normal were screened for inhibitors.

Validation of the analytical procedures was conducted as follows (Bionalysis Report: STER internal document [4 October 2012]):

- FVII:C assay: complete linearity was observed between $1 \%$ and $259 \%\left(\mathrm{r}^{2}=\right.$ 0.99 ), with an intra-assay CV of $1.9-2.6 \%$ and an inter-assay CV of $45-56 \%$.

- Stability of FVII:C at 37\%: no loss of FVII:C was observed, but there was a small increment in activity from hours 1-6. 
- External control: during 12 External Quality Control for Assays and Tests (ECAT), quarterly exercises showed a minimal deviation from the mean of $117-$ 136 laboratories (z-score \pm 1 in 20 exercises and slightly above1 in 4).

- Inhibitor assay intrinsic variation: using an inhibitor with an expected potency of $1 \mathrm{BU}$, the mean $\pm \mathrm{SD}$ of 31 controls was $0.9 \pm 0.1 \mathrm{BU}$.

The research proposed by the STER Study Group was approved by the Ethics Committee of L'Aquila University (the STER coordinator's institution) and, in parallel, by the respective committees of the participating institutions.

\section{Results}

In total, 312 treatments administered to 225 patients with FVII deficiency were reported to STER. Among these, $78.5 \%$ of treatments were with recombinant activated FVII (rFVIIa; NovoSeven $^{\circledR}$, Novo Nordisk A/S, Denmark), 9.9\% were with plasma-derived (pd)-FVII concentrates (Facteur VIITM LFB, Courtaboef, France, or Provertin-UM TIM3 ${ }^{\mathrm{TM}}$, BaxterImmuno, Vienna, Austria), 9.6\% used fresh frozen plasma (FFP), 1.6\% were with prothrombin complex concentrates (PCCs; Prothromplex T TM , Baxter-Immuno, Vienna, Austria) and $0.3 \%$ were with pd-aPCC (FEIBA ${ }^{\mathrm{TM}}$, Baxter-Immuno, Vienna, Austria).

Overall, 115 paired samples suitable for screening were received by the central laboratories. Only those samples with FVII:C levels $<4 \%$ were considered for the inhibitor assay. Samples screened between baseline and day 30 showed that 3/115 (2.6\%) individuals were inhibitor positive. One was a de novo inhibitor (first sample negative, second sample positive; patient 1), and two were 'pre-existing' inhibitors (both samples positive; patients 2 and 4) (Table I). All three of these patients had low FVII:C levels and had previously been exposed to replacement therapy with at least one product (rFVIIa, pd-FVII or FFP). In one patient with a history of repeated bleeds and treatments, an inhibitor was detected in adulthood after a surgical intervention (multiple dental extractions; patient 1), while inhibitors occurred in the other two patients after prolonged rFVIIa prophylaxis for central nervous system (CNS) or gastrointestinal (GI) bleeds (patients 2 [Tokgoz et al, 2012] and 4, respectively) (Table I). An inhibitor was also detected in a patient who had been screened locally for logistical reasons (patient 3); this inhibitor was detected during rFVIIa prophylaxis for CNS bleeds. The three patients who developed inhibitors following 
prophylaxis before the age of 6 months (patients 2, 3 and 4) all had severe clinical phenotypes; however, treatments were continued without apparent loss of efficacy. No anaphylactoid reactions or renal complications were observed during or after prophylaxis.

Clinical inhibitor-related characteristics are shown in Table I. Overall, maximum inhibitor titres varied from 10.4 to $72 \mathrm{BU}$. The occurrence of the first three inhibitors has been reported in previously published studies from the STER (Batorova et al, 2007, Mariani et al, 2011; Mariani et al, 2012b; Mariani et al, 2013; Napolitano et al, 2013).

Data on mutations in the FVII gene were available for two individuals: a missense mutation plus one codon deletion (Ala294Val + Del C) (Batorova et al, 2007) and a homozygous nonsense mutation (p.Ser112-Stop) (Tokgoz et al, 2012) were detected in patients 1 and 2, respectively (Table I).

The inhibitor time course for patient 1 over 6 years is illustrated in Fig 1. Patient 1 was also evaluated kinetically over 24 hours when two different inhibitor titres were present (Fig 2). At the highest inhibitor titre, the area under the curve (AUC) for FVII was approximately 100 times lower, and the clearance approximately 70 times higher, than in patients with FVII deficiency but without inhibitors (unpublished data from STER).

\section{Discussion}

We performed a prospective study of FVII inhibitor occurrence in a large number of patients with congenital FVII deficiency who had previously received replacement therapy for spontaneous or traumatic bleeding episodes (Mariani et al, 2013), for major or minor surgical interventions (Mariani et al, 2011; Mariani et al, 2012b), or for prophylaxis (Napolitano et al, 2013). All inhibitors that developed were high-titre, although very high titres ( $>100 \mathrm{BU})$ were never recorded during follow-up. The method used to detect FVII inhibitors was a modified Bethesda Assay (Ingerslev et al, 2005), with very reproducible analytical features. Although it was not possible to screen all patients of the registry, the prospective study by protocol collected 115 sets of plasma samples, enabling analysis of the incidence of inhibitors following sufficient significant exposure to FVII product..

With regard to the samples tested in the central laboratories, inhibitor development was detected in 2.6\% (3/115) of patients; if all inhibitors observed during the 8-year STER were included, the prevalence was $1.7 \%$ (4/225). Among patients enrolled according to 
protocol, the incidence of de novo inhibitors was $0.8 \%(1 / 115)$. Thus, in this rare bleeding disorder, the development of FVII inhibitors is as rare as that reported in patients with haemophilia B (inhibitor prevalence of 2-3\%) (DiMichele 2007; Astermark et al, 2008; Mariani et al, 2012a). Comparison of FVII deficiency with haemophilia B is plausible given the similar gene and protein structures of FVII and FIX. There are some differences, however; for example, while severe gene defects (such as large or total deletions) have not been reported in patients with FVII deficiency (Mariani et al, 2005), they have been found in patients with haemophilia B with inhibitors. In addition, the immune response in FVII deficiency is apparently not complicated by anaphylactoid reactions, a severe clinical issue in patients with haemophilia B (Chitlur et al, 2009; Mariani et al, 2012a). The absence of this clinical complication in FVII deficiency was confirmed in the present study, in which prophylaxis was continued without any safety concerns in the presence of inhibitors in three patients (patients 2, 3 and 4) (Table I) (Napolitano et al, 2013).

The decrease in FVII inhibitors over time, as demonstrated by patient 1 (Fig 1), is comparable to the time course for FVIII and FIX inhibitors and displays sharp anamnesis following replacement therapy. The kinetics of FVII investigated in this patient in the presence of different inhibitor titres ( 0,4 and $20 \mathrm{BU}$ ) (Fig 2) also confirmed the presence of a strong, titre-dependent, inhibiting activity. In the presence of the highest inhibitor titre, AUC was about 100 times lower and the clearance about 70 times faster than in FVIIdeficient patients without an inhibitor (STER unpublished data).

As described in patients with haemophilia A or B (Christophe et al, 2001; Astermark et al, 2008; Mariani et al, 2012a), the immune reaction in FVII deficiency displays a restricted heterogeneity, with a clear-cut prevalence for the immunoglobulin G4 subclass, although with some variability related to the sample tested .

In the present study, three inhibitors were detected before the age of 6 months (Table I) in patients with a very severe bleeding phenotype; all of these patients received FFP before prophylactic rFVIIa was instituted in response to life-threatening CNS or GI bleeds. The fourth inhibitor developed in patient 1 , who had a history of numerous severe gynaecological bleeds and repeated treatments with FFP, PCC, pd-FVII and rFVIIa. The inhibitor appeared after a surgical intervention covered by rFVIIa; in total, nine bolus injections were administered in 3 days. Considering the clinical treatment histories, no 
clear-cut conclusions can be drawn concerning which replacement therapy actually triggered the immune response.

Gene mutations, evaluable for only two patients in our study (patients 1 and 2), account for low to very low FVII levels and severe clinical phenotypes, especially in the presence of a nonsense mutation (as in patient 2, who had CNS bleeding at 3 months of age). With regard to the frequent p.A354V-p.P464Hfs $\uparrow$ mutation, no other inhibitors have been reported in the combined International Registry on Factor VII Deficiency (IRF7)/STER genetic databases, which contain data on homozygous (12) or compound heterozygous (64) genotypes. Consequently, this peculiar, frequent mutation probably does not represent a high risk for FVII inhibitor development. In contrast to haemophilia B with inhibitors (Mariani et al, 2012a; Collins et al, 2013), FVII deficiency has not been associated with large or complete gene deletions, and nor is the immune response complicated by rare but life-threatening anaphylactoid reactions or renal complications (Chitlur et al, 2009).

Investigators who reported the occurrence of an inhibitor to STER noted that prophylaxis with rFVIIa may still be efficacious (Napolitano et al, 2013). This supports a previous observation of patients with severe FVII deficiency and a very short FVII half-life ( $<1$ hour) who clearly benefited from prophylaxis with rFVIIa (Mathijssen et al, 2004 Tokgoz et al, 2012). These observations may be explained by the rapid clearance of rFVIIa from the circulation through binding to extravascular tissue factor (Hoffman et al, 2007; Gopalakrishnan et al, 2010), as this could exert a protective effect from inhibitory antibodies.

In conclusion, FVII inhibitors in patients with FVII deficiency represent a rare complication ( $\sim 2 \%$ of treated patients), with biochemical and kinetic features similar to those reported in patients with haemophilia A or B. Knowledge of the occurrence and characteristics of inhibitors to FVII is of great interest because this is a rare event in a rare disorder, and an increased understanding of inhibitor development may improve patient management and treatment outcomes.

\section{Acknowledgements}


STER was supported by Novo Nordisk. GM and MP also wish to acknowledge AIFA grant Progetto RF-null-2008-1235892.

$\mathrm{AB}, \mathrm{KK}$ and $\mathrm{UC}$ have received financial support from Novo Nordisk for participation at and/or travel to scientific meetings and congresses. GM has acted as a consultant to, and participated in workshops on behalf of, Novo Nordisk; serves as Coordinator of the International FVII Study Group and STER; has received financial support from various Institutional Research Organisations; and has received unrestricted funding from Novo Nordisk, administered by the Department of Internal Medicine at the University of L'Aquila. ARS has served as a speaker for Novo Nordisk. BS was previously employed as the Director of the Haemostasis Research Unit which received unrestricted research support from Novo Nordisk, Grifols, CSL Behring, LFB, Baxter, SOBI, Bayer and Octapharma; from May 2012 BS has been an employee of Baxter. MK, MP, MN and AD declare no competing financial interests.

The technical support of Dr. Agatha Malefora (St. Thomas Hospital, London UK) is gratefully acknowledged.

$\mathrm{AB}, \mathrm{KK}, \mathrm{UC}$ and $\mathrm{MN}$ performed the research. $\mathrm{AB}, \mathrm{AD}$ and $\mathrm{BS}$ designed the research study. MN was involved in enrolling patients. ARS, KK, UC, MP, AD and BS were involved in data acquisition, analysis, and/or interpretation. GM, MP, AD and BS wrote the paper. KK, ARS, UC, MP, AD and BS critically revised the manuscript. All authors approved the submitted and final versions of the manuscript.

Editorial assistance to the authors during the preparation of this manuscript was provided by Anne Stirland (medical writer, PAREXEL) and financially supported by Novo Nordisk A/S, in compliance with international guidelines for good publication practice. 
Table I. Demographic and clinical data for patients with FVII deficiency with inhibitors.

\begin{tabular}{|c|c|c|c|c|}
\hline \multirow[t]{2}{*}{ Variable } & \multicolumn{4}{|c|}{ Patient number } \\
\hline & 1 & 2 & 3 & 4 \\
\hline Gender/age & Female/53 years & Male/5 years & Male/5 months & Male/1 month \\
\hline Weight & $55.0 \mathrm{~kg}$ & $20.0 \mathrm{~kg}$ & $6.5 \mathrm{~kg}$ & $9.1 \mathrm{~kg}$ \\
\hline Age at inhibitor screening & 59 years & 3 months & 5 months & 1 month \\
\hline FVIIc level & $<1 \%$ & $2 \%$ & $1.3 \%$ & $<1 \%$ \\
\hline FVII gene mutation & p.A354V-p.P464Hfs $\dagger$ & $\begin{array}{l}\text { p.Ser112-Stop } \\
\text { (homozygous) }\end{array}$ & NA & NA \\
\hline First symptom/age at first symptom & Epistaxis/3 years & $\mathrm{CNS} / 3$ months & CNS and GI/birth & Tongue haematoma/1 month \\
\hline Symptoms reported ${ }^{*}$ & Br-Ep-Gum-Hp-Me & CNS-Br-Ep-GI-Gum & CNS-GI-Gum & CNS-Br-Ep-Gum-Mu-Sc \\
\hline Previous treatments & FFP, pd-FVII, rFVIIa & FFP & FFP & FFP \\
\hline Event at inhibitor discovery & Multiple dental extraction & Prophylaxis (CNS) & Prophylaxis (CNS) & Prophylaxis (GI) \\
\hline Pre-inhibitor treatment ${ }^{\dagger}$ & rFVIIa & rFVIIa & rFVIIa & rFVIIa \\
\hline Schedule & $\begin{array}{c}\text { Initial bolus } 30 \mu \mathrm{g} / \mathrm{kg} \text { plus } \\
8 \text { consecutive boluses } 10 \mu \mathrm{g} / \mathrm{kg}\end{array}$ & $30 \mu \mathrm{g} / \mathrm{kg} \times 3$ weeks & $65 \mu \mathrm{g} / \mathrm{kg} \times 1$ week & $31 \mu \mathrm{g} / \mathrm{kg} \times 3$ weeks \\
\hline Inhibitor titres, minimum-maximum & $10-20 \mathrm{BU}$ & $38-68.3 \mathrm{BU}$ & $5.5-60 \mathrm{BU}$ & $32-72 \mathrm{BU}$ \\
\hline Concomitant medications & Tamoxifen, antifibrinolytics & None & Ciprofloxacin and fluconazole & None \\
\hline
\end{tabular}

*Br, easy bruising; Ep, epistaxis; Gum, gum bleeding; Me, menorrhagia; Hp, haemoperitoneum; CNS, central nervous system bleeding; GI, gastrointestinal bleeding; Mu, muscle haematoma; Sc, subcutaneous haematoma; FFP, fresh frozen plasma; pd-FVII, plasma-derived FVII concentrate; rFVIIa, recombinant activated FVII.

${ }^{\dagger}$ Treatment upon inhibitor discovery. 
Fig 1. The time course of the inhibitor titre and the effect of re-exposure to FVII in inhibitor patient 1 with severe FVII deficiency (baseline FVIIc, $0.8 \mathrm{IU} / \mathrm{dl}$ ). Arrows indicate administration of pd-FVII and rFVIIa.

[Ideally, fig to be formatted so that: pd-FVII not pdFVIII; $x$-axis legend lower case; use $m l($ not $m L)]$

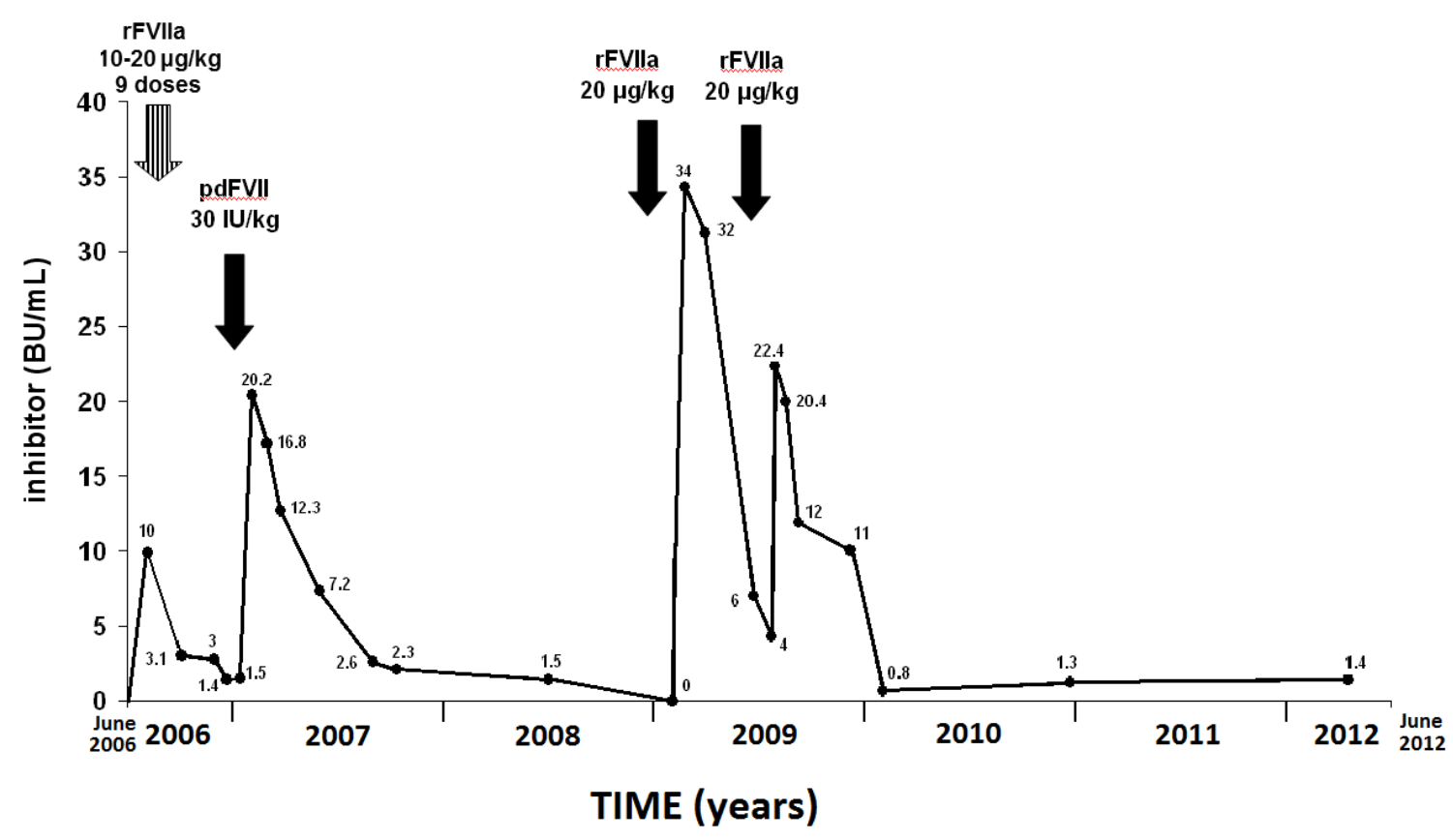


Fig 2. Pharmacokinetics of FVIIc after rFVIIa $20 \mu \mathrm{g} / \mathrm{kg}$ in a non-inhibitor patient with severe FVII deficiency and in Patient 1 with inhibitor, investigated at two occasions at different inhibitor levels (4.0 BU/ml and 20.4 BU/ml).

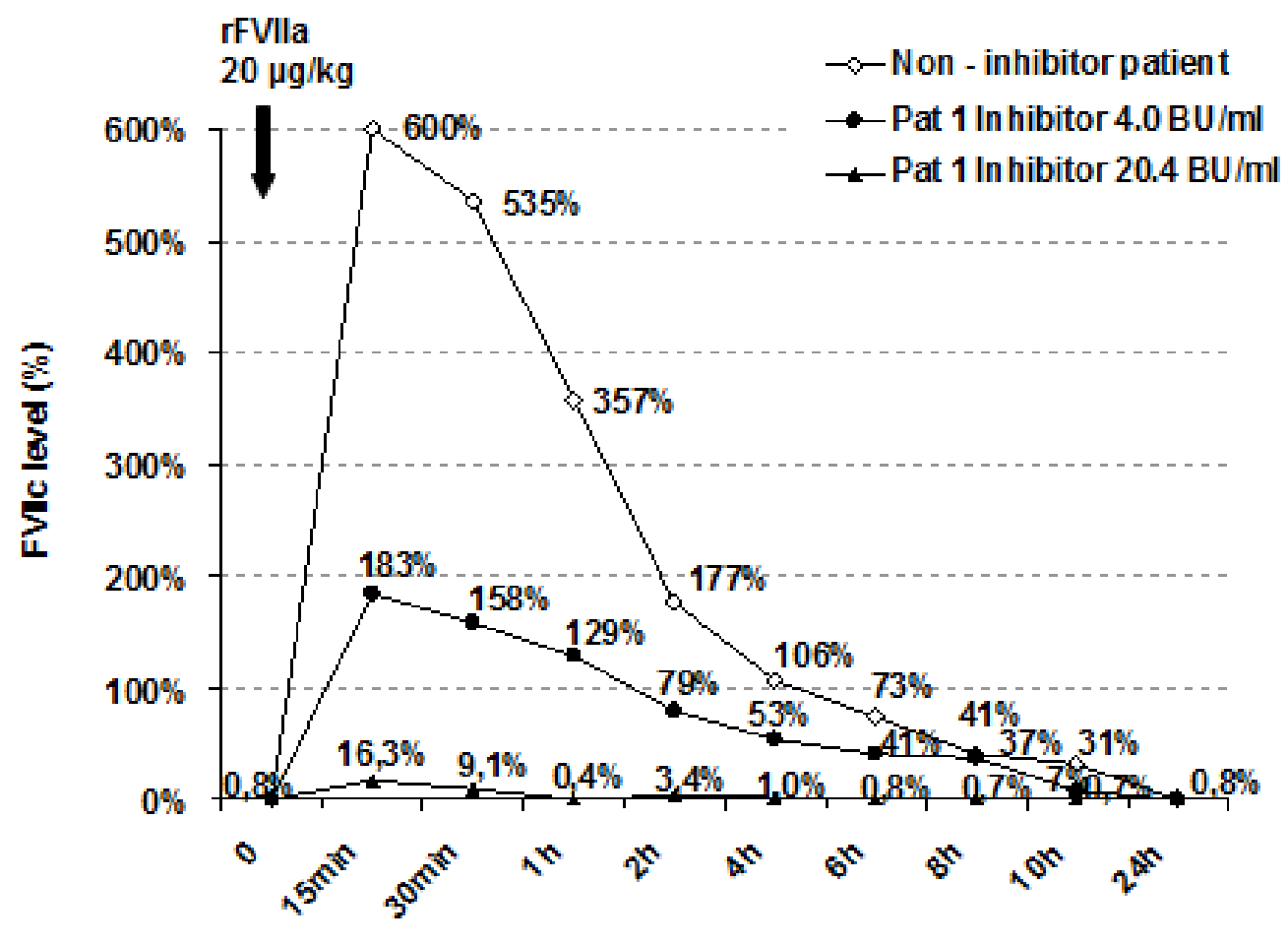

Time 


\section{References}

Astermark,J., Lacroix-Desmazes, S. \& Reding, M.T. (2008) Inhibitor development. Haemophilia, 14 (Suppl. 3), 36-42.

Batorova, A., Mariani, G., Ingerslev, J., Schved, J. \& Auerswald, G., Herrmann, F., Balazova, E., on behalf of the IRF7SG. (2007) Inhibitor of FVII in congenital severe factor VII deficiency. Journal of Thrombosis and Haemostasis, 5 (Suppl. 2), P-S-199.

Bernardi, F., Dolce, A., Pinotti, M., Shapiro, A.D., Santagostino, E., Peyvandi, F., Batorova, A., Lapecorella, M., Schved, J.F., Ingerslev, J. \& Mariani, G. (2009) Major differences in bleeding symptoms between factor VII deficiency and hemophilia B. Journal of Thrombosis and Haemostasis, 7, 774-779.

Bolton-Maggs, P.H. (2006) Optimal haemophilia care versus the reality. British Journal of Haematology, 132, 671-682.

Chitlur, M., Warrier, I., Raipurkar, M. \& Lusher, J.M. (2009) Inhibitors in factor IX deficiency a report of the ISTH-SSC international FIX inhibitor registry (1997-2006). Haemophilia, 15, 1027-1031

Christophe, O.D., Lenting, P.J., Cherel, G., Boon-Spijker, M., Lavergne, J.M., Boertjes, R., Briquel, M.E., de Goede-Bolder, A., Goudemand, J., Gaillard, S., d'Oiron, R., Meyer, D. \& Mertens, K. (2001) Functional mapping of anti-factor IX inhibitors developed in patients with severe hemophilia B. Blood, 98, 1416-1423.

Collins, P.W., Chalmers, E., Hart, D.P., Liesner, R., Rangarajan, S., Talks, K., Williams, M., Hay, C.R. \& UK Haemophilia Centre Doctors (2013) Diagnosis and treatment of factor VIII and IX inhibitors in congenital haemophilia: (4th edition). UK Haemophilia Centre Doctors Organization. British Journal of Haematology, 160, 153-170. 
Coppola, A., Santoro, C., Tagliaferri, A., Franchini, M. \& Di Minno, G. (2010)

Understanding inhibitor development in haemophilia A: towards clinical prediction and prevention strategies. Haemophilia, 16 (Suppl. 1), 13-19.

DiMichele, D. (2007) Inhibitor development in haemophilia B: an orphan disease in need of attention. British Journal of Haematology, 138, 305-315

DiMichele, D.M. (2012) Immune tolerance in haemophilia: the long journey to the fork in the road. British Journal of Haematology, 159, 123-134.

Franchini, M., Frattini, F., Crestani, S. \& Bonfanti, C. (2013) Alloantibodies in previously untreated hemophilia A patients: the role of environmental factors. Hematology, 2013 18, 183-90.

Furie, B. \& Furie, B.C. (1992) Molecular and cellular biology of blood coagulation. New England Journal of Medicine, 326, 800-806.

Gopalakrishnan, R., Hedner, U., Ghosh, S., Nayak, R.C., Allen, T.C., Pendurthi, U.R. \& Rao, L.V. (2010) Bio-distribution of pharmacologically administered recombinant factor VIIa (rFVIIa). Journal of Thrombosis and Haemostasis, 8, 301-310.

Hoffman, M., Colina, C.M., McDonald, A.G., Arepally, G.M., Pedersen, L. \& Monroe, D.M. (2007) Tissue factor around dermal vessels has bound factor VII in the absence of injury. Journal of Thrombosis and Haemostasis, 5, 1403-1408.

Ingerslev, J., Christiansen, K. \& Sorensen, B. (2005) Inhibitor to factor VII in severe factor VII deficiency: detection and course of the inhibitory response. Journal of Thrombosis and Haemostasis, 3, 799-800.

Lawrence, J.S. \& Johnson, J.B. (1941) The presence of a circulating anti-coagulant in a male member of a hemophiliac family. Transactions of the American Clinical and Climatological Association, 57, 223-231. 
Mariani, G. \& Bernardi, F. (2009) Factor VII deficiency. Seminars in Thrombosis and Hemostasis, 35, 400-406.

Mariani, G., Testa, M.G., Di Paolantonio, T., Molskov Bech, R. \& Hedner, U. (1999) Use of recombinant, activated factor VII in the treatment of congenital factor VII deficiencies. Vox Sanguinis, 77, 131-136.

Mariani, G., Herrmann, F.H., Dolce, A., Batorova, A., Etro, D., Peyvandi, F., Wulff, K., Schved, J.F., Auerswald, G., Ingerslev, J. \& Bernardi, F. (2005) Clinical phenotypes and factor VII genotype in congenital factor VII deficiency. Thrombosis and Haemostasis, 93, 481-487.

Mariani, G., Dolce, A., Batorova, A., Auerswald, G., Schved, J.F., Siragusa, S., Napolitano, M., Knudsen, J.B. \& Ingerslev, J. (2011) Recombinant, activated factor VII for surgery in factor VII deficiency: a prospective evaluation - the surgical STER. British Journal of Haematology, 152, 340-346.

Mariani, G., Konkle, B. \& Kessler, C.M. (2012a) Inhibitors in Hemophilia A and B. In Hematology. Basic Principles and Practice. Hoffman R, Benz EJ, Silberstein LE, Heslop HE, Weitz JI, Anastasi J. Elsevier. Philadelphia PA, USA. Chapter 138.

Mariani, G., Dolce, A., Napolitano, M., Ingerslev, J., Giansily-Blaizot, M., Di Minno, M.D., Auerswald, G., De Saez, A.R., Tagliaferri, A. \& Batorova, A. (2012b) Invasive procedures and minor surgery in factor VII deficiency. Haemophilia, 18, e63-e65.

Mariani, G., Napolitano, M., Dolce, A., Perez, G.R., Batorova, A., Karimi, M., Platokouki, H., Auerswald, G., Bertrand, A.M., Di Minno, G., Schved, J.F., Bjerre, J., Ingerslev, J., Sorensen, B. \& Ruiz-Saez, A; Seven Treatment Evaluation Registry; International Factor VII Deficiency Study Group. (2013) Replacement therapy for bleeding episodes in factor VII deficiency. A prospective evaluation. Thrombosis and Haemostasis, 109, 238-247.

Mathijssen, N.C., Masereeuw, R., Verbeek, K., Lavergne, J.M., Costa, J.M., van Heerde, W.L. \& Novakova, I.R. (2004) Prophylactic effect of recombinant factor VIIa in factor VII deficient patients. British Journal of Haematology, 125, 494-499. 
Napolitano, M., Giansily-Blaizot, M., Dolce, A., Schved, J.F., Auerswald, G., Ingerslev, J., Bjerre, J., Altisent, C., Charoenkwan, P., Michaels, L., Chuansumrit, A., Di Minno, G., Caliskan, U. \& Mariani, G. (2013) Prophylaxis in congenital factor VII deficiency: indications, efficacy and safety. Results from the Seven Treatment Evaluation Registry (STER). Haematologica, 98, 538-544.

Nicolaisen, E.M., Hansen, L.L., Poulsen, F., Glazer, S., Hedner, U. (1996)

Immunological aspects of recombinant factor VIIa (rFVIIa) in clinical use. Thromb

Haemost, 76, 200-204.

Oldenburg, J. \& Pavlova, A. (2006) Genetic risk factors for inhibitors to factors VIII and IX. Haemophilia, 12 (Suppl. 6), 15-22.

Pruthi, R.K., Rodriguez, V., Allen, C., Slaby, J.A., Schmidt, K.A. \& Plumhoff, E.A. (2007) Molecular analysis in a patient with severe factor VII deficiency and an inhibitor: report of a novel mutation (S103G). European Journal of Haematology, 79, 354-359.

Tokgoz, H., Caliskan, U., Lavigne-Lissalde, G., \& Giansily-Blaizot, M. (2012) Successful prophylactic use of recombinant activated factor VII (rFVIIa) in a patient with congenital FVII deficiency and inhibitors to FVII. Haemophilia, 18, e25-e27. 